\title{
Contribuciones de la neurociencia al entendimiento de la creatividad humana
}

\section{Contributions of neuroscience to the understanding of human creativity}

\author{
FRANCISCO-J. RoDRÍGUEZ-MuÑOZ \\ Universidad de Almería \\ frodriguez@ual.es
}

Recibido: 9 de febrero de 2011

Aprobado: 22 de marzo de 2011

\begin{abstract}
Resumen
La creatividad y el proceso creativo son conceptos complejos que han recibido gran atención durante todas las épocas y desde diversos campos del conocimiento. En la actualidad, ha sido incipiente el interés que algunas disciplinas científicas, como es el caso de la neurociencia, han mostrado por contribuir al entendimiento de este proceso cognitivo que tiene evidentes correlatos en el funcionamiento del cerebro humano. El objetivo de este artículo es revisar algunos de los principales hallazgos que se han producido en la investigación neurocientífica durante las últimas décadas. De este modo, se discuten las principales regiones nerviosas que pueden estar involucradas en el proceso de la creatividad, en el bloqueo creativo, y su relación con otras variables como la inteligencia o los sueños. Finalmente, estos progresos suponen, sin lugar a dudas, una contribución clave para la comprensión del ser humano y de su comportamiento en sociedad.
\end{abstract}

Palabras Clave: creatividad, proceso creativo, cerebro, neurociencia.

Rodríguez-Muñoz, F-J. 2011: Contribuciones de la neurociencia al entendimiento de la creatividad humana. Arte, Individuo y Sociedad, 23 (2), 45-54

\begin{abstract}
Creativity and the creative process are complex concepts that have received much attention at all times and from different fields of knowledge. Nowadays, some scientific disciplines, including neuroscience, have contributed substantially to the explanation of this cognitive process that has clear correlates in the human brain structure. The aim of this paper is to review some key findings that have occurred in neuroscience research in recent decades. It is also discussed the main nerve regions that might be involved in the process of creativity, in the creative block, and its relationship to other variables such as intelligence or dreams. Finally, these developments imply, without doubt, an important contribution to the understanding of human nature and its influence on social behaviour. Key Words: creativity, creative process, brain, neuroscience.
\end{abstract}

Rodríguez-Muñoz, F-J. 2011: Contributions of neuroscience to the understanding of human creativity. Arte, Individuo y Sociedad, 23 (2), 45-54

Sumario: 1. Introducción, 2. Neurociencia y neurofisiología de la dimensión creativa, 2.1 Bloqueo creativo, 2.2 Condicionantes de la creatividad, 3. A modo de conclusión. Agradecimientos y financiación. Referencias 


\section{Introducción}

La creatividad ha sido objeto de estudio por parte de filósofos, sociólogos, psicólogos, pedagogos $\mathrm{y}$, más contemporáneamente, han ido surgiendo interesantes aportaciones en el campo de la investigación neurocientífica que han supuesto un considerable avance en la comprensión de este acto humano.

A pesar de todas las formulaciones que ha recibido a lo largo de la historia, la noción de creatividad -tal y como aquí la concebimos- hace referencia a la capacidad que poseen las personas para producir ideas originales y, a partir de estas, originar materiales nuevos, tomando siempre como referente el contexto social en el que tienen ocasión esas innovaciones -las cuales, en definitiva, comportan la expansión de los campos conceptuales y estéticos preexistentes-. Si bien es verdad que la creatividad puede ser estudiada desde múltiples ámbitos del conocimiento, el arte constituye un espacio propicio y sin igual para su desarrollo y análisis.

Entre las denominaciones que se han utilizado como sinónimas de la creatividad humana, las más habituales han sido inventiva, originalidad, imaginación constructiva o pensamiento divergente. Destacamos, por su carácter sintético, la aproximación conceptual de Corbalán-Berná (2008) para quien la creatividad es entendida como la capacidad de utilizar conocimientos e informaciones de forma novedosa, y de hallar soluciones divergentes a los problemas. Según Rouquette (1973), han sido cuatro las asociaciones principales que los teóricos han establecido al intentar definir la creatividad: (1) como un "don divino"; (2) como un producto; (3) como un rasgo de la personalidad; y (4) como un proceso. De acuerdo con Chávez (2001), entendemos que la creatividad es un proceso que consiste en generar algo -conceptual, material o estético- nuevo y/o transformador.

Otra de las cuestiones que no ha estado exenta de debates es la que concierne a las etapas por las que transcurre todo proceso creativo. Entre los primeros modelos, el de Wallas (1926) insiste en cinco periodos que vienen determinados por la preparación, la incubación, la intimación, la iluminación y la verificación de la idea creativa. En efecto, para Bogousslavsky (2005) la propuesta clásica responde a cuatro fases sucesivas que quedarían sintetizadas en la preparación, la incubación, la inspiración y la producción de la inventiva.

Según Chávez (2001), este proceso se desarrolla en tres etapas fundamentales: (1) asociación-integración: los sujetos realizan asociaciones entre el mundo externo y su mundo interno, y toman conciencia de ellas; (2) elaboración: el sujeto parte de estas combinaciones para producir su obra valiéndose de su propio talento; y (3) comunicación: el individuo hace pública su creación y transmite su interioridad a los sujetos receptores sobre los que produce nuevas percepciones. Estas tres fases pueden dar lugar a una retroalimentación, siendo entonces los sujetos receptores quienes se convierten en creadores a partir del nuevo mensaje creativo que han recibido.

Como plantea Runco (2004), vivimos en un mundo cambiante y cada vez más complejo en el que la creatividad implica una reacción a los problemas y a los desafíos del futuro; por tanto, esta capacidad -innovadora, transformadora y constructiva- se convierte en uno de los motores de la evolución cultural. Trazando un paralelismo con el materialismo dialéctico, este autor relaciona la formación del pensamiento divergente con un periodo previo en el que existe una perspectiva individual (o 
tesis), un movimiento hacia las alternativas originales que presenta esa perspectiva (o antítesis) y, por último, una integración (o síntesis) entre ambos estados. Asimismo, han sido varias las discusiones académicas motivadas en torno a las consideraciones que debe reunir el sujeto creativo, poniendo a veces el acento en los procesos cognitivos que subyacen a esta capacidad -el procesamiento de información compleja, la percepción o la imaginación-; otras en la influencia del ambiente (Batey y Furnham, 2006); y en las últimas décadas se ha ido incorporando el examen de la estructura, de las funciones químicas y de las interacciones que suceden en el sistema nervioso y que, en última instancia, son las responsables de las conductas creativas (Jung et al., 2010).

El estudio de la creatividad es un reto para la investigación neurobiológica, ya que implica la integración de diversas funciones mentales y de todos los componentes individuales que están relacionados con la experiencia propia. Sin embrago, este hecho podría transformar la visión que tenemos de nosotros mismos y de nuestras modernas sociedades (Chávez et al., 2004; Zeki, 2001).

Está bastante extendida la creencia de que los pacientes psiquiátricos son poseedores de un elevado potencial creativo (Bogousslavsky, 2005). Por ejemplo, tras la figura de Van Gogh siempre pesará el mito negro del esquizofrénico que un día se amputó una oreja, ingresó en un sanatorio y, posteriormente, se pegó un tiro. Un número considerable de los artistas expresionistas neoyorquinos -activos en la década de los cuarenta y hasta los años sesenta- sufrieron psicopatologías emocionales, trastornos depresivos, alcoholismo y protagonizaron varios intentos de suicidio, a pesar de sus incuestionables habilidades para la creación. En esta misma línea, Joan Miró es una muestra más de cómo un estado de ánimo depresivo e inestable, con fluctuaciones hacia la bipolaridad, puede estar ligado a una enorme capacidad creativa (Schildkraut y Otero, 1996). De hecho, muchos de los esfuerzos por detallar el funcionamiento neurológico de la capacidad creativa se han basado tradicionalmente en datos procedentes de pacientes psiquiátricos (Pollack, Mulvenna y Lythgoe, 2007). No obstante, hay que ser prudentes al interpretar estos ejemplos $\mathrm{y}$ hemos de procurar no caer en falsas generalizaciones, pues parece razonable que la creatividad exija un mínimo de organización y de coherencia mental.

Nuestro objetivo en este artículo es revisar las evidencias más recientes que han aparecido, durante la última década, en las investigaciones neurocientíficas y neurofisiológicas que han prestado atención a la creatividad en el ser humano. El panorama en el que vivimos reclama una mayor comunicación entre las investigaciones científicas y los procesos creativos. Según creemos, las técnicas de estudio que emplea la neurociencia pueden ser de gran utilidad para resolver nuestras incógnitas acerca del funcionamiento del cerebro y, de este modo, pueden acelerar nuestro conocimiento acerca del ser humano y de su comportamiento social.

\section{Neurociencia y neurofisiología de la dimensión creativa}

Los hallazgos neurocientíficos sobre la creatividad en los últimos años, por lo general, han sido compatibles con muchas de las herramientas y pruebas de evaluación que proporciona la neuropsicología -desde diversos enfoques-, vinculando el comportamiento al funcionamiento del sistema nervioso humano. Siguiendo a 
Dietrich (2007), uno de los principales retos, en ese sentido, ha sido evitar caer en visiones más o menos reduccionistas a partir de la presentación de constructos cognitivos que pretenden explicar los procesos creativos. Al mismo tiempo, uno de los objetivos de la neurociencia se precisa en la síntesis teórica del gran número de metodologías y técnicas que actualmente han surgido para valorar y dar respuesta a la expresión de la creatividad.

La producción creativa ha sido analizada a través de numerosas actividades que guardan una estrecha relación con el arte (Jung et al., 2010); por ejemplo, la improvisación musical (Bengtsson, Csikszentmihalyi y Ullen, 2007; Berkowitz y Ansari, 2008; Brown, Martínez y Parsons, 2006; Limb y Braun, 2008); el baile (Fink, Graif y Veubauer, 2009); o la percepción y la estética visual (Bhattacharya y Petsche, 2002; Kirk et al., 2009), por citar solo algunos trabajos que nos parecen relevantes.

Los avances neurológicos sobre la creatividad han tendido a centrarse en la implicación mayor de los lóbulos frontales o de otras regiones más posteriores del cerebro durante el proceso de creación (Heilman, Nadeau y Beversdorf, 2003) e, incluso, algunos han planteado la predominancia de estructuras subcorticales -como los ganglios basales, asociados al control de los movimientos- (Dietrich, 2004) a la hora de ofrecer explicaciones neurológicas sobre esta capacidad humana. Por otro lado, Jung et al. (2010) sugieren que existe una distribución significativa de las áreas cerebrales durante el proceso creativo y sostienen que el flujo de información -a través de estas regiones- es clave para nuestro entendimiento de la neurofisiología de la originalidad y la inventiva. Esta es también una de las conclusiones principales a las que llegan Jung y Haier (2007) en un estudio previo sobre la inteligencia, basándose en distintos procedimientos de neuroimagen estructural y funcional.

En la actualidad es ampliamente asumido que el cerebro funciona como un todo y tiene una importante función globalizadora. Además, no hay dudas sobre el papel principal que tiene el córtex cerebral y, más concretamente, el córtex prefrontal, donde residen las bases neuronales responsables de las funciones cognitivas superiores (Fuster, 2002). Esta región es la que está involucrada en el desarrollo de las funciones ejecutivas y, por lo tanto, es la encargada de procesar la información compleja que llega al cerebro, buscar soluciones, formular planes, proponer estrategias para un comportamiento social acorde con el contexto en el que se produce, y finalmente dar instrucciones al córtex motor para la ejecución de esas acciones. El córtex prefrontal, como es bien sabido, es la última estructura cerebral en desarrollarse filogenética y ontogénicamente, y en los seres humanos no alcanza un grado de madurez apropiado hasta aproximadamente los veinte años (Fuster, 2000). Como indica Dietrich (2004), la producción de ideas y acciones novedosas es frecuente en el procesamiento cognitivo de la información.

Partiendo de estas premisas, nos interesa referirnos al modelo que propone Flaherty (2005) sobre la generación de ideas creativas. Para esta autora, las conexiones entre los lóbulos frontales y los lóbulos temporales son más importantes que las interacciones que mantienen el hemisferio izquierdo y el derecho en el cerebro -este último popularmente denominado cerebro creativo-. Además, asigna un papel preponderante al sistema límbico -encargado de gestionar las respuestas fisiológicas ante los estímulos emocionales- durante el proceso en el que tiene lugar la generación de nuevas 
ideas. En este esquema, la dopamina facilita las actividades voluntarias y orientadas a un único objetivo, a la vez que inhibe los comportamientos competitivos.

\subsection{El bloqueo creativo}

En un estudio anterior, Flaherty (2004) vincula las disfunciones del lóbulo frontal con el bloqueo creativo - esto es, la falta de ideas originales-. La depresión es un trastorno que está asociado, precisamente, a un funcionamiento anómalo de esta región cerebral y, a menudo, se manifiesta en una falta acusada de motivación y flexibilidad cognitiva. De hecho, cuando la depresión es tratada clínicamente, se ha observado que las funciones del lóbulo frontal se normalizan en las pruebas de imagen funcional (Goldapple et al., 2004).

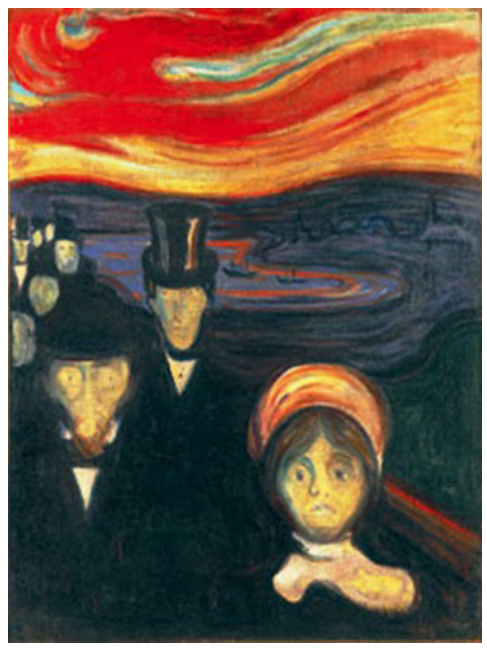

Figura 1. Ansiedad, Munch, 1894. Los rostros pálidos, los gestos crispados o el ambiente distorsionado son elementos creativos que sugieren agitación y desasosiego en el sujeto receptor.

Asimismo, la ansiedad ha sido vinculada al bloqueo creativo y, como la depresión, conlleva una serie de cambios en el funcionamiento del lóbulo frontal (Cannistraro y Rauch, 2003). Estudios electromagnéticos como el de Carlsson, Wendt y Risberg (2000) han demostrado que esta región del cerebro humano es susceptible de ser estimulada por medio de tareas que demandan creatividad.

La falta de ideas creativas, según Flaherty (2004), también podría estar ligada a determinadas perturbaciones en el funcionamiento del lóbulo frontal:

El año pasado, sin ir más lejos, (...) quedé trágicamente bloqueado como escritor tras publicar "Nada más jamás", mi libro sobre los escritores que renuncian a escribir. Pasé bastantes meses sin ideas para un nuevo libro, como si estuviera recibiendo un castigo por haber escrito sobre los que dejan de escribir. (...) Escribia sobre lo que fuera con tal de no sentirme totalmente bloqueado (Vila-Matas, 2002). 
Autores como Chávez et al. (2004) han constatado que los índices de creatividad se encuentran en sintonía con un mayor flujo cerebral en las áreas que se relacionan con el procesamiento multimodal y de emociones, y concluyen que la creatividad es un proceso dinámico que implica la integración de todos estos procesos, realizándose en un sistema muy distribuido en el cerebro.

\subsection{Condicionantes de la creatividad}

Las correlaciones entre la creatividad y la inteligencia han dado lugar a puntos de vista opuestos en la actualidad. Para Jiménez et al. (2008), en el terreno de la psicopedagogía, los superdotados y los talentos creativos se caracterizan por poseer una mayor creatividad en relación con otras personas que no presentan altas capacidades intelectuales. Del mismo modo, para estos autores el perfil del superdotado está más asociado a la dimensión figurativa de la creatividad, mientras que el talento creativo muestra una mayor creatividad verbal. Para Boden (1998), la creatividad es también un signo fundamental del comportamiento inteligente en el ser humano. Sin embargo, otras perspectivas, como la de Kalbfleisch (2004), sostienen que la creatividad precisa cocientes intelectuales mínimos y, en consecuencia, tener talento no equivale siempre a capacidades intelectuales elevadas.

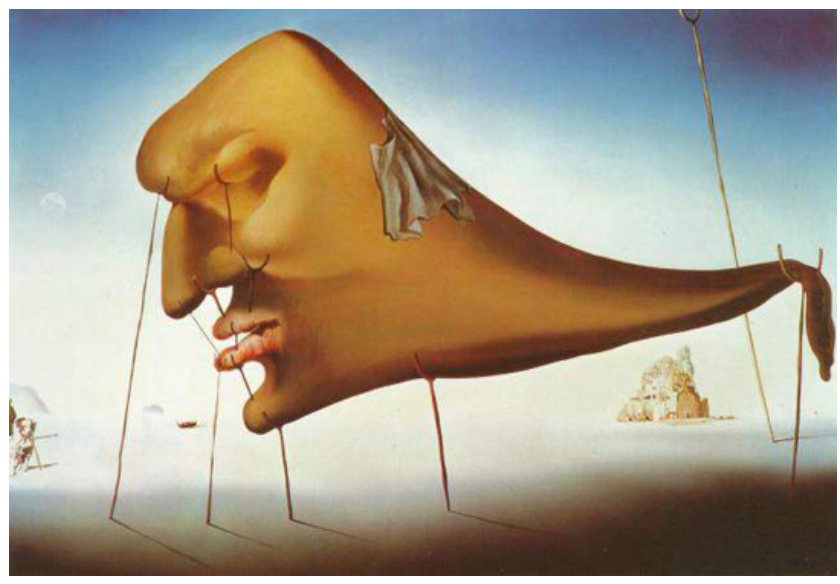

Figura 2. El sueño, Dalí, 1937. El óleo representa uno de los temas más fascinantes para los surrealistas: el mundo de los sueños; reveladores de la actividad subconsciente y detonadores de la fuerza creadora.

Los sueños han sido una constante creativa en todas las épocas artísticas. El papel del sueño y de las revelaciones, en los procesos de producción artística, ha sido relacionado con ciertos mecanismos de desinhibición cerebral que favorecen la creatividad (Bogousslavsky, 2002; Stickgold y Walker, 2005); así pues, algunos trabajos concluyen que el descanso, la relajación, la meditación y los sueños son una fuente inmejorable de ideas creativas (Dietrich, 2004). Por ejemplo, Paul MacCartney confesó haber encontrado el famoso tema musical Yesterday en uno de sus sueños. 
Frankenstein, al parecer, también surgió de la inspiración a partir de un sueño -o tal vez de una pesadilla- que tuvo Mary Shelley; algo similar ha revelado Stephen King sobre las ideas que destilan sus novelas de terror. De acuerdo con las palabras del psicólogo alemán Otto Loewi, los argumentos que le permitieron probar que los impulsos nerviosos dependían de una transmisión química -hallazgo que le valió el Premio Nobel de Medicina en 1936- llegaron a su mente mientras dormía.

En otro orden de cosas, estudios como el de Razumnikova (2004) encuentran diferentes patrones de conectividad interhemisférica entre hombres y mujeres, señalando divergencias significativas -motivadas por el sexo- en la neurobiología de la creatividad. Asimismo, no podemos perder de vista la enorme cantidad de técnicas de neuroimagen que se han aplicado al estudio neurobiológico de la capacidad creativa, entre las que destacan las de flujo sanguíneo cerebral regional (rCBF), la resonancia magnética funcional (fMRI), la tomografía por emisión de positrones (PET), o la tomografía computerizada por emisión de fotón único (SPECT).

\section{A modo de conclusión}

Este breve repaso sobre los avances en la investigación neurocientífica ha puesto en evidencia que, en los últimos años, ha existido un notable interés por desarrollar teorías y aplicar los nuevos métodos de registro de la actividad cerebral y cartografiado anatómico que, en definitiva, han contribuido a un mayor entendimiento del fenómeno de la creatividad humana.

En primer lugar, parece existir un consenso generalizado en cuanto a que esta capacidad, como proceso cognitivo complejo, requiere la intervención de varios circuitos cerebrales, localizados en ambos hemisferios. Por tanto, no consiste en la activación de un solo hemisferio -el hemisferio derecho o creativo, como habitualmente se pensaba-, sino que se trata de un proceso distribuido globalmente en el cerebro.

Durante el proceso creativo parece fundamental la interacción que se produce entre los lóbulos frontales y los temporales en el cerebro. Asimismo, el sistema límbico ocupa un papel principal en las respuestas a los estímulos emocionales, y la dopamina es uno de los neurotransmisores que facilita las actividades relacionadas con la creatividad, según sugieren estudios como el de Flaherty (2005). Por otro lado, se ha comprobado que el bloqueo creativo -o la falta de ideas creativas- está ligado a algunos tipos de disfunciones en el lóbulo frontal; esto es, las áreas cerebrales que están implicadas dejan de activarse con la misma intensidad. Trastornos como la depresión o la ansiedad guardan también una estrecha relación con las perturbaciones que pueden originarse en esta región cerebral (Flaherty, 2004).

Además, diversos autores están de acuerdo en que los circuitos que participan en la producción de conceptos altamente novedosos y originales son similares a los que generan ideas comunes. De este modo, habremos de tener en cuenta factores individuales y de percepción social a la hora de evaluar más específicamente esta capacidad. Por ejemplo, todavía es preciso ahondar en las correlaciones que, desde diversos puntos de vista, se establecen entre la inteligencia y la creatividad. Desde otra perspectiva, resulta atractivo el estudio neurocientífico que hace corresponder la fase de iluminación o inspiración, en el proceso creativo, con el estado de ensoñación. 
Finalmente, estamos convencidos de que la neurociencia está abriendo nuevos horizontes para el estudio de la creatividad y, por esta razón, creemos que serán decisivos los hallazgos que realizará en torno a este proceso cognitivo, muestra extraordinaria de las repercusiones que tiene el procesamiento de la información en el cerebro. Estos avances, en todo caso, nos ayudarán a entender mejor la naturaleza y el comportamiento social del ser humano.

\section{Agradecimientos y financiación}

Este trabajo está dedicado al Grupo de Investigación "Neuropsicología de la Atención" (CTS-135) de la Universidad de Almería, dirigido por la doctora $\mathrm{M}^{\mathrm{a}}$ Ángeles Fernández Estévez, y ha sido realizado gracias al apoyo del Programa FPU del Ministerio de Educación (Gobierno de España).

\section{Referencias}

Batey, M. y Furnham, A. (2006). Creativity, intelligence, and personality: A critical review of the scattered literature. Genetic, Social, and General Psychology Monographs, 132, 355-429.

Bengtsson, S. L., Csikszentmihalyi, M., Ullen, F. (2007). Cortical regions involved in the generation of musical structures during improvisation in pianists. Journal of Cognitive Neuroscience, 19, 830-842.

Berkowitz, A. L. y Ansari, D. (2008). Generation of novel motor sequences: The neural correlates of musical improvisation. Neuroimage, 41, 535-543.

Bhattacharya, J. y Petsche, H. (2002). Shadows of artistry: Cortical synchrony during perception and imagery of visual art. Brain research. Cognitive brain research, $13,179-186$.

Boden, M. A. (1998). Creativity and artificial intelligence. Artificial Intelligence, $103,347-356$.

Bogousslavsky, J. (2002). Le rêve est-il une seconde vie? Médecine et Hygiene, 2390, 875-876.

Bogousslavsky, J. (2005). Artistic creativity, style and brain disorders. European Neurology, 54, 103-111.

Brown, S., Martínez, M. J. y Parsons, L. M. (2006). Music and language side by side in the brain: A PET study of the generation of melodies and sentences. European Journal of Neuroscience, 23, 2791-2803.

Cannistraro, P. A. y Rauch, S. L. (2003). Neural circuitry of anxiety: evidence from structural and functional neuroimaging studies. Psychopharmacology Bulletin, $37,8-25$.

Carlsson, I., Wendt, P. E. y Risberg, J. (2000). On the neurobiology of creativity. Differences in frontal activity between high and low creative subjects. Neuropsychologia, 38, 873-885.

Chávez, R. A. (2001). Evaluación de la relación entre creatividad, personalidad y psicopatología. México: UNAM. 
Chávez, R. A., Graff-Guerrero, A., García-Reyna, J. C., Vaugier, V. y Cruz-Fuentes, C. (2004). Neurobiología de la creatividad: resultados preliminares de un estudio de activación cerebral. Salud Mental, 27, 38-46.

Corbalán-Berná, F. J. (2008). ¿De qué se habla cuando hablamos de creatividad? Cuadernos FHyCS-UNJu, 35, 11-21.

Dietrich, A. (2004). The cognitive neuroscience of creativity. Psychonomic Bulletin and Review, 11, 1011-1026.

Dietrich, A. (2007). Who's afraid of a cognitive neuroscience of creativity? Methods, 42, 22-27.

Fink, A., Graif, B., Veubauer, A. C. (2009). Brain correlates underlying creative thinking: EEG alpha activity in professional vs. novice dancers. Neuroimage, 46, 854-862.

Flaherty, A. W. (2004). The midnight disease: The drive to write, writer's block, and the creative brain. Boston: Houghton Mifflin.

Flaherty, A. W. (2005). Frontotemporal and dopaminergic control of idea generation and creative drive. The Journal of Comparative Neurology, 493, 147-153.

Fuster, J. M. (2000). The prefrontal cortex of the primate: A synopsis. Psychobiology, $28,125-131$.

Fuster, J.M.(2002). Frontal lobe and cognitive development. Journal of Neurocytology, 31, 373-385.

Goldapple, K., Segal, Z., Garson, C., Lau, M., Bieling, P., Kennedy, S. y Mayberg, H. (2004). Modulation of corticallimbic pathways in major depression: treatmentspecific effects of cognitive behavior therapy. Archives of General Psychiatry, $61,34-41$.

Heilman, K. M., Nadeau, S. E. y Beversdorf, D. O. (2003). Creative innovation: Possible brain mechanisms. Neurocase, 9, 369-379.

Jiménez, J. E., Artiles, C., Rodríguez, C., García, E., Camacho, J. y Moraes, J. (2008). Creatividad e inteligencia: ¿dos hermanas gemelas inseparables? Revista Española de Pedagogía, 240, 261-282.

Jung, R. E. y Haier, R. J. (2007). The Parieto-Frontal Integration Theory (P-FIT) of intelligence: Converging neuroimaging evidence. Behavioral and Brain Sciences, 30, 135-154.

Jung, R. E., Segall, J. M., Bockholt, H. J., Flores, R. A., Smith, S. M., Chávez, R. S. y Haier, R. J. (2010). Neuroanatomy of creativity. Human Brain Mapping, 31, 398-409.

Kalbfleisch, M. L. (2004). The functional neural anatomy of talent. The Anatomical Record, 277B, 21-36.

Kirk, U., Skov, M., Hulme, O., Christensen, M. S. y Zeki, S. (2009). Modulation of aesthetic value by semantic context: An fMRI study. Neuroimage, 44, 1125-1132.

Limb, C. J. y Braun, A. R. (2008). Neural substrates of spontaneous musical performance: An FMRI study of jazz improvisation. PLoS ONE, 3, e1679.

Pollack, T. A., Mulvenna, C. M. y Lythgoe, M. F. (2007). De novo artistic behaviour following brain injury. En Bogousslavsky, J. y Hennerici, M. G. (eds.), Neurological Disorders in Famous Artists, vol. 2 (pp. 75-88). Basel: Karger. 
Razumnikova, O. M. (2004). Gender differences in hemispheric organization during divergent thinking: An EEG investigation in human subjects. Neuroscience Letters, 362, 193-195.

Rouquette, M. (1973). La Créativité. París: PUF.

Runco, M. A. (2004). Creativity. Annual Review of Psychology, 55, 657-687.

Schildkraut, J. J. y Otero, A. (1996). Depression and the spiritual in modern art. Chichester: Wiley.

Stickgold, R. y Walker, M. (2004). To sleep, perchance to gain creative insight? Trends in Cognitive Sciences, 8, 191-192.

Vila-Matas, E. (2002). El mal de Montano. Barcelona: Anagrama.

Wallas, G. (1926). The art of thought. Nueva York: Harcourt Brace.

Zeki, S. (2001). Artistic creativity and the brain. Science, 293, 51-52. 